\title{
EXCLUDED VOLUME EFFECTS AND BINARY CLUSTER INTEGRALS IN DILUTE POLYMER SOLUTIONS
}

\author{
HiRomi YamaKawa \\ Department of Polymer Chemistry, Kyoto University, Kyoto, Japan
}

\begin{abstract}
Recent advances in the theory of dilute polymer solutions are critically summarized. The following topics are discussed: theory of the excluded volume effect, including perturbation theory, the asymptotic solution, and approximate treatments; the second virial coefficient; the theory of intrinsic viscosity, especially perturbation theory: comparison of theory with experiment using data recently obtained for monodisperse polystyrenes; methods of determining the binary cluster integral: and simple analysis of the binary cluster integral by a cell-theory approach.
\end{abstract}

\section{INTRODUCTION}

The statistical mechanics of polymer solutions has been for many years one of the active fields of polymer science because of its close relation to molecular characterization. Specifically, since the concept of the excluded volume effect in a polymer chain was introduced by Flory ${ }^{1,2}$ in 1949, there have been a number of significant advances in the study of this effect and the related equilibrium and nonequilibrium properties of dilute polymer solutions. These advances, made during the last two decades, have delineated the relationship between the theory in the field, now called the two-parameter theory, and other branches of the molecular sciences. There are now various aspects of the field which are covered by the two-parameter theory ${ }^{3}$. In this paper, emphasis is focused on the following characteristics and problems.

First, the excluded volume problem in a single polymer chain is a manybody problem, and many mathematical difficulties are encountered. Indeed, an exact asymptotic solution for an infinitely long chain with large excluded volume has not yet been obtained, though the problem may be unravelled applying the techniques used in solving many-body problems in simple fluids and many-electron systems. This is in contrast with the problem in configurational statistics of polymer chains without excluded volume which is equivalent to the problem in one-dimensional cooperative spin systems and is therefore amenable to exact solution. Although the asymptotic solution to the excluded volume problem cannot be directly compared with experiment, it is of great statistical-mechanical interest and is also useful in deriving approximate closed expressions. 
Second, there have been a number of attempts to derive approximate closed expressions for the expansion factor and the second virial coefficient which are valid over a wide but experimentally accessible range of excluded volume interaction. However, there have been very few investigations of the nonequilibrium properties, such as intrinsic viscosity, of polymer chains with excluded volume. In this case, we must solve simultaneously the problem of polymer dynamics, such as the problem of the hydrodynamic interaction which exists even in the unperturbed $\Theta$-state. Nevertheless, the establishment of such consistent expressions for all equilibrium and nonequilibrium properties is very important, because it provides an understanding of the physical processes in dilute polymer solutions and also methods of determining the two basic molecular parameters, the unperturbed dimension and the binary cluster integral for a pair of segments. We note that several advances have recently been made also in the perturbation theories of equilibrium and nonequilibrium properties.

Third, when we attempt to make experimental tests of the derived approximate expressions, a fundamental difficulty arises from the fact that the binary cluster integral and hence the excluded volume parameter are not directly observable. Even with Monte Carlo data, another difficulty arises from the fact that there is a gap between lattice chains and polymer chains. This is in contrast with the theory of simple fluids. Although the latter also involves the many-body problem, experimental tests of the derived approximate equations of state can be achieved by examining the relationship between pressure and density with the use of actual experimental data and also of molecular dynamics data.

The fourth point is concerned with the theoretical interpretation of the two basic molecular parameters appearing in the theory. As for the unperturbed molecular dimension, extensive investigations have been made during the past decade, and the methods and results are summarized in books by Volkenstein ${ }^{4}$, by Birshtein and Ptitsyn ${ }^{5}$, and by Flory ${ }^{6}$. The present paper is not, of course, intended to discuss this subject. On the other hand, very few investigations of the binary cluster integral have been published. However, it is this parameter that has a close relation to the excluded volume effect, and plays an important role in the interpretation of interactions in dilute polymer solutions on the molecular and atomic levels.

In the following sections, we discuss these problems in more detail, summarizing the results recently obtained by the present author and collaborators. We emphasize that the resolution of the many-body problem in polymers consists of exploring the dependence of dilute-solution properties on polymer molecular weight, and that this leads to correct estimates of the molecular parameters.

\section{TWO-PARAMETER THEORY}

Consider a model polymer chain composed of $n$ identical segments (beads) joined linearly with a bond of effective length $a$. Suppose that interactions between two segments belonging to the same chain or different chains in dilute solutions may be described in terms of the pair correlation 
function $g(r)$ as a function of the separation $r$ between segments averaged over all orientations. With $g(r)$, define the binary cluster integral $\beta$ by

$$
\beta=\int[1-g(r)] \mathrm{d} r,
$$

which represents the effective excluded volume for a pair of segments at infinite dilution. Then, all the equilibrium and nonequilibrium properties of dilute polymer solutions may be expressed in terms of the two parameter combinations $n a^{2}$ and $n^{2} \beta$ within the framework of the two-parameter theory, i.e., on the assumptions that $n \gg 1, \beta \ll\left(n a^{2}\right)^{\frac{3}{2}}$, and the potential is pairwise additive. We may choose as the two basic parameters the unperturbed mean-square end-to-end distance $\left\langle R^{2}\right\rangle_{0}$ (or the unperturbed meansquare radius of gyration $\left\langle S^{2}\right\rangle_{0}$ ) and the excluded volume parameter $z$ :

$$
\begin{aligned}
& \left\langle R^{2}\right\rangle_{0}=6\left\langle S^{2}\right\rangle_{0}=n a^{2}, \\
& z=\left(3 / 2 \pi\left\langle R^{2}\right\rangle_{0}\right)^{\frac{3}{2}} n^{2} \beta .
\end{aligned}
$$

We note that the parameters $n$ and $a$, and also $n$ and $\beta$, never appear separately in the final equations. In other words, the final equations are invariant to the choice of $n$, and the value of $n$ may therefore be, to some extent, arbitrary as long as the value of $\beta$ itself is not discussed.

\section{Expansion factors}

We define expansion factors $\alpha_{R}$ and $\alpha_{S}$ by

$$
\alpha_{R}^{2}=\left\langle R^{2}\right\rangle /\left\langle R^{2}\right\rangle_{0}
$$

and

$$
\alpha_{S}^{2}=\left\langle S^{2}\right\rangle /\left\langle S^{2}\right\rangle_{0}
$$

with $\left\langle R^{2}\right\rangle$ and $\left\langle S^{2}\right\rangle$ the mean square end-to-end distance and radius of gyration of the chain with excluded volume, respectively. These expansion factors may be expressed as functions of $z$ only. For small $z$, an evaluation of $\alpha_{R}$ and $\alpha_{S}$ can be carried out in a manner similar to the virial or cluster expansion of the gas pressure at low density. The results obtained so far are summarized as follows,

$$
\begin{gathered}
\alpha_{R}^{2}=1+1.333 z-2.075 z^{2}+6.459 z^{3}-\ldots, \\
\alpha_{S}^{2}=1+1.276 z-2.082 z^{2}+\ldots
\end{gathered}
$$

The linear term of $\alpha_{R}{ }^{2}$ was derived by Teramoto ${ }^{7}$ and many other investigators ${ }^{8-11}$ long ago, the quadratic term of $\alpha_{R}{ }^{2}$ and the linear term of $\alpha_{S}{ }^{2}$ being due to Fixman ${ }^{10}$. The cubic term of $\alpha_{R}{ }^{2}$ and the quadratic term of $\alpha_{S}{ }^{2}$ have recently been derived by Yamakawa et al. ${ }^{12,13}$.

The investigation of the other extreme, i.e., the asymptotic solution for $\alpha_{R}$ at large $z$, has been initiated by Edwards ${ }^{14}$, applying the self-consistent field method. Subsequently, this approach has been further investigated by Reiss $^{15}$ and others ${ }^{16,17}$. However, these investigations involve some numerical error and incorrect derivations. The correct integro-differential 
equations for the distribution function have been obtained by Yamakawa ${ }^{18,19}$ and Freed ${ }^{20}$ for both the zeroth-order field of Edwards and the first-order field of Reiss. For the Edwards field, the solution is

$$
\lim _{z \rightarrow \infty} \alpha_{R}^{v}=C z
$$

with $v=5$ and $C=0.744^{14,19}$. (Edwards has erroneously obtained the value of 1.49 for $C$.) For the Reiss field, the exact solution of the integrodifferential equation has not yet been obtained. but it has been solved by Yamakawa ${ }^{18}$ only in a uniform-expansion approximation with the result $v=5$ and $C=1.45$. These recent results support the earlier conclusion of Flory ${ }^{1,2}$. though there has been controversy on the question of whether the behavior of $\alpha_{R}$ or $\alpha_{S}$ obeys an equation of the fifth-power type $(v=5)$ or of the third-power type $(v=3)^{21,22}$. We note that integro-differential equations have also been derived by the liquid theory approach ${ }^{23.24}$.

As for the derivation of approximate closed expressions for $\alpha_{R}$ or $\alpha_{S}$, various attempts have been made for many years ${ }^{3}$. In these the problem has been approached from two starting points. One begins with the closed form of the distribution function of the end-to-end distance $\boldsymbol{R}$.

$$
P(\boldsymbol{R})=Z^{-1} P_{0}(\boldsymbol{R}) \exp [-V(R) / k T],
$$

where $Z$ is the configurational partition function, $P_{0}(R)$ is the unperturbed distribution, and $V(R)$ is the potential of mean force with $R$ fixed. In this approach, the problem is to evaluate $V(R)$. Recently. Fujita et $a l^{25}$ have shown that within the framework of the two-parameter theory. $V(R)$ may be expressed exactly in the form,

$$
V(R)=k T \sum_{i<j} \int_{0}^{\beta} P\left(O_{i j} \mid \boldsymbol{R}\right) \mathrm{d} \beta,
$$

where the conditional probability density, $P\left(O_{i j} \mid \boldsymbol{R}\right)$, for the contact between the $i$ th and $j$ th segments with $\boldsymbol{R}$ lixed is to be evaluated in the perturbed state. In the earlier treatments belonging to this category, the conditional probability density in equation 8 was evaluated in the unperturbed state. However, this has a significant influence on the final result. For instance, Kurata, Stockmayer, and Roig ${ }^{21}$ have evaluated $P$ in equation 8 in the unperturbed state for an ellipsoid model to derive an equation of the third-power type for $\alpha_{R}$, while evaluation of $P$ in the perturbed state for the same model leads to an equation of the fifth-power type ${ }^{25}$. In general, fifth-power type equations are obtained irrespective of the form of distribution of segments if $P$ is evaluated in the perturbed state with the use of a uniform-expansion approximation $^{3,25,26}$. We note that Alexandrowicz ${ }^{27}$ has also used an expression for $\boldsymbol{V}(\boldsymbol{R})$ similar to equation 8 .

There hold similar equations also for the distribution function $P(S)$ of the radius of gyration $S$ and the potential $V(S)$. Strictly, however, $P_{0}(S)$ is not Gaussian, and the problem is more difficult. If we assume the Gaussian $P_{0}(S)$ and the spherically symmetric distribution of segments, that is:

$$
V(S)=C S^{-3} z k T,
$$


and $C$ is adjusted to give the exact first-order perturbation theory, the modified Flory $(F, m)$ equation for $\alpha_{s}$ is obtained. Flory and Fisk $(F F)^{28}$ have improved the form of $P_{0}(S)$ in an approximate fashion to re-evaluate $\alpha_{S}$, based on the investigation of $P_{0}(S)$ by Fixman $^{29}$ and Forsman ${ }^{30,31}$. However, the exact solution for $P_{0}(S)$, though only numerical. has recently been obtained by Koyama ${ }^{32}$ and Fujita ${ }^{33}$. With this result, Fujita and Norisuye $(\mathrm{FN})^{33}$ have revised the calculation of Flory and Fisk. In Figure 1

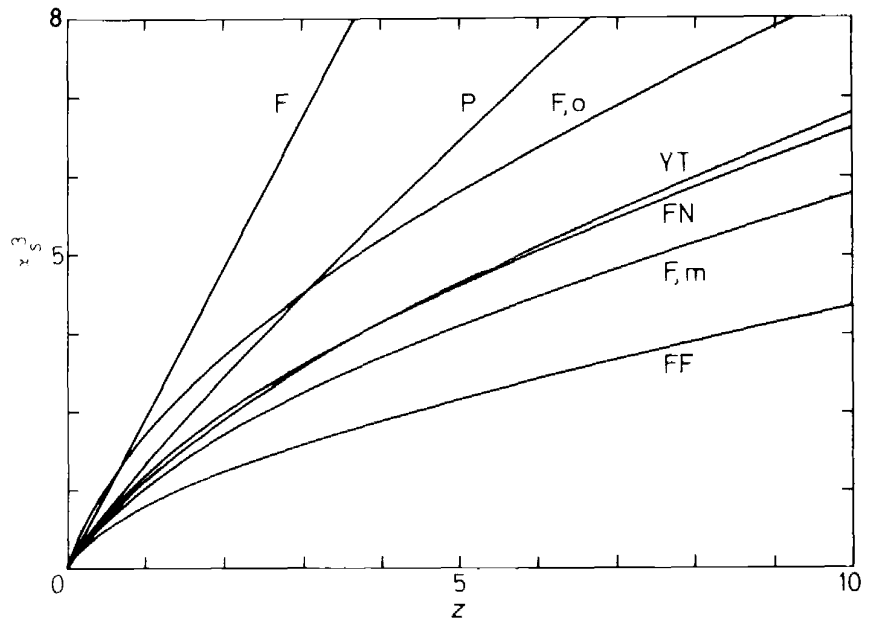

Figure 1. Values of $\alpha_{S}^{3}$ calculated from various approximate theories. Curve F,o: the original Flory theory. Curve F,m: the modified Flory theory. Curve FF: the Flory-Fisk theory. Curve FN: the Fujita-Norisuye theory. Line F: the Fixman theory. Curve P: the Ptitsyn theory. Curve YT: the Yamakawa-Tanaka theory.

are plotted the values of $\alpha_{s}{ }^{3}$ predicted by these three theories. Comparing the three curves in the Figure, it is clear that the improvement of Flory and Fisk has been made in the wrong direction. We note that the recent bosonoperator theory of Fixman ${ }^{34.35}$ also gives values of $\alpha_{S}{ }^{3}$ smaller than those predicted by the F,m theory.

The other approach is a derivation of a differential equation for $\alpha_{R}$ or $\alpha_{S}$. It was attempted first by Fixman $(F)^{10,36}$, and subsequently by Ptitsyn $(\mathrm{P})^{37}$. Both these theories give equations of the third-power type for $\alpha_{R}$ and $\alpha_{S}$. Yamakawa and Tanaka $(\mathrm{YT})^{13}$ have extended this approach to derive a hierarchy of differential equations for $\alpha_{R}$ or $\alpha_{S}$, by analogy with the technique in the theory of liquids. The hierarchy has been truncated appropriately to give higher-order approximations to $\alpha_{R}$ and $\alpha_{S}$. The values of $\alpha_{S}{ }^{3}$ predicted by these three theories are also plotted in Figure 1. It is seen that the values predicted by the YT theory are very close to those predicted by the FN theory of the fifth-power type. In the Figure are also plotted the values predicted by the original Flory $(\mathrm{F}, \mathrm{o})$ theory ${ }^{1}$. 
Although it is difficult to test these approv:..nate expressions for $\alpha_{R}$ or $\alpha_{S}$ as functions of $z$ using Monte Carlo data ${ }^{3}$, the recent available data ${ }^{38-40}$ lead to the definite conclusion that neither $\alpha_{R}{ }^{3}$ nor $\alpha_{S}{ }^{3}$ is linear in $n^{\frac{1}{2}}$. Further, the extrapolated data for lattice chains ${ }^{41-43}$ support the asymptotic solution of the fifth-power type.

\section{Second virial coefficients}

The second virial coefficient $A_{2}$ may be expressed in the form.

$$
A_{2}=4 \pi^{\frac{3}{2}} N_{A}\left(\left\langle S^{2}\right\rangle^{\frac{3}{2}} / M^{2}\right) \Psi \text {. }
$$

where $N_{A}$ is the Avogadro number. $M$ is the polymer molecular weight, and $\Psi$ is a function of $z$ which represents the degree of interpenctration of polymer molecules in dilute solution. The problem is to evaluate $\Psi$. and this is also a many-body problem. For small $z$, the cluster-expansion technique is again useful. The results obtained so far are summarized as follows,

$$
\Psi=\bar{z}\left(1-2.865 \bar{z}+9.202 \bar{z}^{2}-\ldots\right)
$$

with $\bar{z}=z / x_{S}{ }^{3}$. In equation 10 , we have ignored the correlation between intramolecular and intermolecular interactions. since it has no great influence on the result ${ }^{44.45}$. The linear term in the parentheses of equation 10) has been derived by $\mathrm{Zimm}^{46}$ and others ${ }^{47.48}$, and the Albrecht value ${ }^{4 / 7}$ of 9.726 for the coefficient of the quadratic term has recently been corrected by Tagami and Casassa ${ }^{45}$ as above.

The asymptotic solution for $\Psi$ at large $\bar{z}$ has not yet been investigated. However. from a simple physical consideration, it may be expected that in the limit $z \rightarrow x$, polymer molecules behave like rigid spheres in dilute solutions, and therefore $\Psi$ becomes a constant independent of $z$.

On the other hand. various approximate closed expressions for $\Psi$ have been derived ${ }^{3}$. Then the following question arises: what expression for $\alpha_{S}$ is to be combined with a given expression for $\Psi$ in order to complete the form of $\Psi$ as a function of $z$ or $\alpha_{s}$. Necessarily this must be done from the point of view of maintaining the self-consistence of the intramolecular and intermolecular theories. First, the Kurata- Yamakawa (KY) equation ${ }^{3.44 .49}$ for $\Psi$ may be combined with the Yamakawa-Tanaka (YT) equation ${ }^{13}$ for $\alpha_{\mathrm{S}}$ :

$$
\begin{array}{rlrl}
\Psi & =0.547\left[1-(1+3.903 \bar{z})^{-0.4683}\right], & & (\mathrm{KY}) \\
\alpha_{\mathrm{S}}{ }^{2}=0.541+0.459(1+6.04 z)^{0.46} . & & (\mathrm{YT})
\end{array}
$$

The reason is that both have been derived by the hierarchy approach, and the intramolecular and intermolecular hierarchies have been truncated in mathematically consistent closure approximations. The pair of equations 11 and 12 is referred to as combination $Y$. There are two other possible, though not completely justifiable, combinations. One is the combination (F.o) of the original Flory-Krigbaum-Orofino (FKO,o) equation ${ }^{50.51}$ for $\psi$ and the original Flory $(\mathrm{F}, \mathrm{o})$ equation ${ }^{1}$ for $\alpha_{S}$. The other is the combination (F.m) of the modified Flory-Krigbaum Orofino (FKO,m) equation ${ }^{50}{ }^{52}$ for $\Psi$ and the modified Flory $(F, m)$ equation for $\alpha_{s}$. In these the intramolecular and intermolecular theories have been derived on the basis of the smoothed 
EXCLUDED VOLUME EFFECTS AND BINARY CLUSTER INTEGRALS

Gaussian density model. However, there is no explicit justification of any combination.

\section{Intrinsic viscosities}

There are two problems in the development of the theory of intrinsic viscosities $[\eta]$. One is concerned with the unperturbed chain, that is, the evaluation of the Flory-Fox constant $\Phi_{0}$ (in the unperturbed state) ${ }^{53}$, and the other is to evaluate the viscosity-radius expansion factor $\alpha_{\eta}$ defined by

$$
\begin{aligned}
{[\eta] } & =6^{\frac{3}{2}} \Phi_{0}\left(\left\langle S^{2}\right\rangle_{0}^{\frac{3}{2}} / M\right) \alpha_{\eta}{ }^{3} \\
& =6^{\frac{3}{2}} \Phi\left\langle S^{2}\right\rangle^{\frac{3}{2}} / M .
\end{aligned}
$$

It is important to note that the evaluation of $\alpha_{\eta}$ depends on the theory of $\Phi_{0}$.

Many investigations of $\Phi_{0}$, and more generally the dynamical properties of dilute polymer solutions, have been published ${ }^{3}$. These have two common foundations. One is the use of the Oseen hydrodynamic interaction tensor introduced first by Kirkwood and Riseman ${ }^{54}$, and ti:o other is the reduction to diffusion problems in the chain configuration space formulated first by Kirkwood $^{55}$ in a very general form and later by Rouse ${ }^{56}$, Bueche ${ }^{57}$, and Zimm ${ }^{58}$ more conveniently using normal coordinates. Recently, the use of the Oseen formula has been criticized by De Wames, Hall, and Shen ${ }^{59}$ and by Zwanzig, Kiefer, and Weiss ${ }^{60}$. In particular, the latter authors have shown that in the Oseen approximation, with beads (segments) treated as point sources of friction, the frictional forces exerted by the beads on the fluid can become singular for some values of the strength of the hydrodynamic interaction. Perico and Rossi ${ }^{61}$ and Thurston and Morrison ${ }^{62}$ have also encountered a similar situation in the calculation of the intrinsic viscosity of short chains. The difficulty may be eliminated by finite bead models. Such investigations have been very recently initiated by Rotne and $\operatorname{Prager}^{63}$ and by Yamakawa ${ }^{64-66}$. However, it seems that the Oseen formula has still some practical value in the case of flexible chains.

There is another difficulty concerning the hydrodynamic interaction. Use of the Oseen formula gives the draining effect, that is, the dependence of $\Phi_{0}$ on molecular weight. However, such an effect has never been observed experimentally for flexible chain polymers. Very little attention has been directed to this problem ${ }^{64.67 .68}$, and a complete solution has not yet been obtained. It is interesting to note that according to the recent calculation of Edwards and Oliver ${ }^{69}$ the draining effect does not occur in the frictional coefficient of a flexible cylinder. In the following discussion, we assume that the theory for impermeable molecules based on the Oseen formula are valid for molecular weights of ordinary interest. In Table 1 are summarized the values of $\Phi_{0}$ derived in the nondraining limit by Kirkwood, Riseman, Auer, and Gardner $(\mathrm{KRAG})^{54,70}$, by Zimm $(\mathrm{Z})^{58}$, by Hearst $(\mathrm{H})^{71}$, and by Fixman and Pyun (FP) ${ }^{72.73}$. The Hearst theory does not differ from the Zimm theory except that the Rouse eigenfunctions are adopted for all values of the draining parameter. The Oseen tensor is pre-averaged in the first three theories of the Table, while in the Fixman-Pyun theory this is avoided by a perturbation method. Thus, an exact value of $\Phi_{0}$ has not been derived, its best experimental value being $2.5 \times 10^{23}(\mathrm{cgs})$. 


\begin{tabular}{|c|c|}
\hline Theory & $\Phi_{0} \times 10^{.23} \mathrm{cgs}$ \\
\hline $\mathrm{KR} A \mathrm{G}$ & 2.87 \\
\hline Z & 2.84 \\
\hline $\mathrm{H}$ & 2.82 \\
\hline $\mathrm{FP}(0$ th order $)$ & 2.68 \\
\hline (1st order) & 1.81 \\
\hline (2nd order) & 2.66 \\
\hline
\end{tabular}

Now. $\alpha_{\eta}$ becomes a function of $z$ alone in the nondraining limit. At small $z, \alpha_{\eta}{ }^{3}$ may be expanded in the form.

$$
\alpha_{\eta}{ }^{3}=1+C_{1} z-\cdots .
$$

According to the semi-empirical theory of Flory and Fox ${ }^{53} \cdot \alpha_{\eta}{ }^{3}$ is equal to $\alpha_{s}{ }^{3}$, so that $C_{1}=1.914$. Kurata and Yamakawa ${ }^{48}$ have introduced approximately the excluded volume effect into the Kirkwood-Riseman theory and obtained the value of 1.55 for $C_{1}$. Fixman ${ }^{34}$ has introduced the effect into the Hearst theory by an application of the boson-operational technique and obtained the value of 1.80 for $C_{1}{ }^{i 4}$. However, his procedure is still approximate. Very recently, Yamakawa and Tanaka ${ }^{74}$ have introduced exactly the effect into the Hearst theory on the basis of the Fixman-Pyun theory and obtained the value of 1.06 for $C_{1}$. At present, this last value may be regarded as most rigorous.

However, there has not been derived an approximate closed expression for $\alpha_{\eta}$ which gives the first-order perturbation theory of Yamakawa and Tanaka at small $z$.

\section{COMPARISON WITH EXPERIMENT}

Great efforts have been made to achieve a direct experimental test of the two-parameter theory, instead of an indirect test provided by viscosity plots $^{22,75}$. The foremost of these is the work of Berry ${ }^{74.77}$. As already mentioned, the binary cluster integral $\beta$, and hence the parameter $z$, cannot be estimated directly from experiment. However, Berry has attempted to estimate $\beta$, assuming the temperature dependence,

$$
\beta=\beta_{0}(1-\Theta / T),
$$

where $\beta_{0}$ is a constant independent of temperature, and can be determined from the temperature dependence of $A_{2}$ near the $\Theta$-temperature. Thus Berry has determined values of $z$ for polystyrene in decalin and toluene, and plotted $\alpha_{s}{ }^{2}$, and also $\alpha_{\eta}{ }^{3}$, against $z$. We believe that it is the best way to use, if possible, these plots as a criterion of validity for a theory. However, there is no justification of validity of the assumption (15) over a wide range of temperature $^{49}$, and moreover, it is difficult to determine $\beta_{0}$, accurately. 
Subsequently, experimental tests have therefore been attempted without the use of any ad hoc assumption for polychloroprene by Norisuye et al. ${ }^{78.79}$, for poly-p-methylstyrene by Tanaka et al. ${ }^{80}$, and for poly-p-bromostyrene by Takashima et $a l .^{81}$. In this paper, we use light-scattering and viscosity data obtained recently by our group ${ }^{82}$ for monodisperse polystyrenes (prepared anionically in tetrahydrofuran) to test the theory following our procedure.

\section{Expansion factors and second virial coefficients}

What have been adopted as criteria of validity of a theory are the following: (1) consistency in the values of $z$ determined from observed values of $\alpha_{S}$ and from observed values of $\Psi$ using the theoretical expressions for $\alpha_{S}$ and $\Psi$, and (2) linearity between $M^{\frac{1}{2}}$ and $z$ determined from observed values of $\alpha_{S}$ using its theoretical expression. The first criterion arises from the fact that the binary cluster integral for segments in the same chain must be the same as that for two segments belonging to different chains. Clearly the second criterion is required by the definition of $z$. These two criteria involve no assumptions.

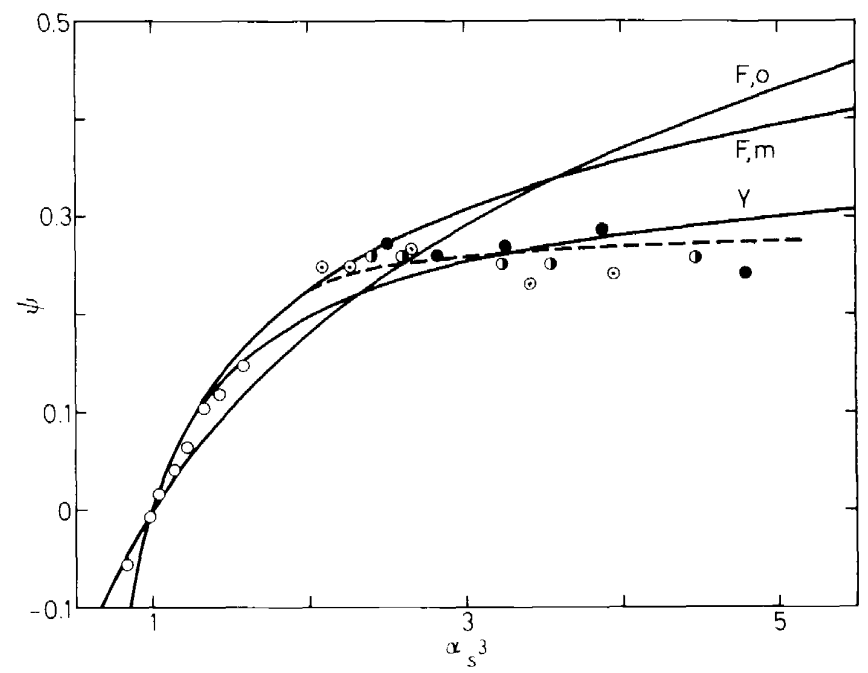

Figure 2. Plots of $\Psi$ against $\alpha_{S}{ }^{3}$ for polystyrene: in benzene at $30 \mathrm{C}:($ in toluene at $30 \mathrm{C}$ : $\odot$ in dichloroethane at $30 \mathrm{C}: 0$. in cyclohexane at various temperatures ${ }^{82}$. The broken curve is an empirical fit to the previous data. The full curves represent the theoretical values calculated from the three combinations of the theories of $\psi$ and $\alpha_{S}$ defined in the text.

The fulfilment of criterion (1) may be examined conveniently by constructing plots of $\Psi$ against $\alpha_{S}{ }^{3}$, as proposed by Fujita et al. ${ }^{78}$. Figure 2 shows such plots for polystyrene in benzene, toluene, and dichloroethane at $30^{\circ} \mathrm{C}$ and in cyclohexane at temperatures ranging from $32^{\circ}$ to $60^{\circ} \mathrm{C}^{82}$. The data for $A_{2}$ and $\left\langle S^{2}\right\rangle$ have been obtained from light-scattering measurements by the method of square-root plots ${ }^{76}$. The broken curve represents the 
best fit to all published data cited above, the present data being consistent with those. Thus both $\Psi$ and $\alpha_{s}$ are seen to be functions of $z$ only. The three full curves in the figure represent the values calculated from combinations $\mathrm{F}, \mathrm{o}, \mathrm{F}, \mathrm{m}$, and $\mathrm{Y}$ of intramolecular and intermolecular theories. It is seen that combination $\mathrm{Y}$ is most satisfactory. Strictly, this combination, and also the other two, cannot explain the experimental curve which is almost horizontal for $\alpha_{s}{ }^{3}>2$. The Casassa-Markovitz theory ${ }^{83}$ can explain such a trend. but cannot predict values of $\Psi$ greater than 0.2 , this being in disagreement with experiment.

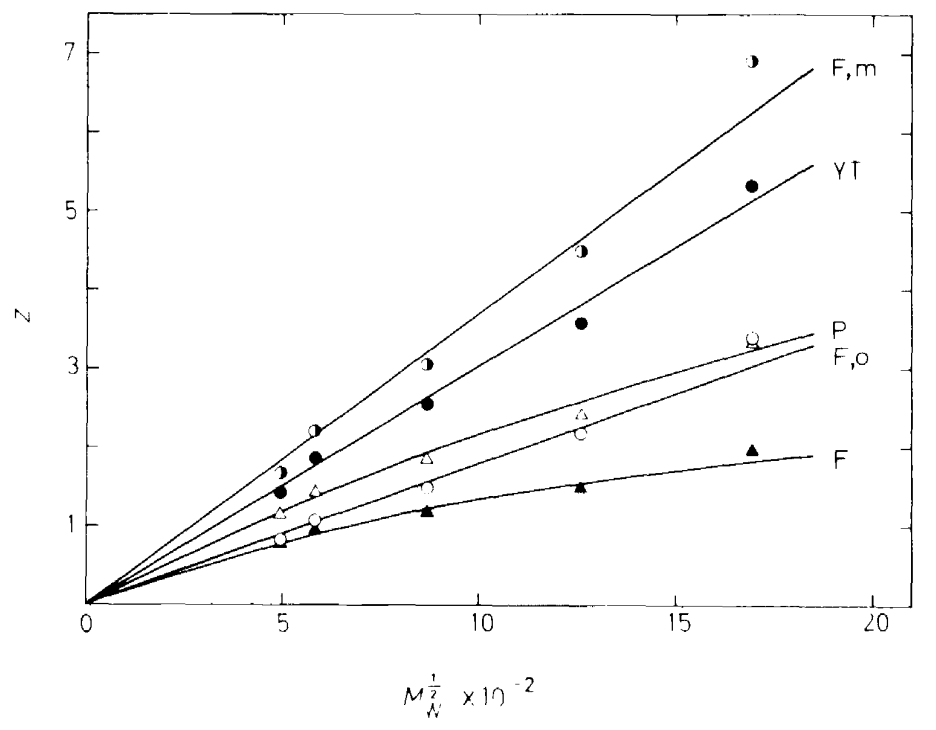

Figure 3 . Test of the linearity between $z$ and $M_{w^{2}}{ }^{1}$ with the data for polystyrene in benzene at 3) $\mathrm{C}^{82}$. The values of z were calculated from various theories of $x_{5}$. from the f.o theory:

D. Irom the F.m theory, from the YT theory. $\boldsymbol{A}$. from the F theory: $\triangle$. from the P theory.

We now turn to the examination of criterion (2). Figure 3 shows plots of $z$ against the square-root of the weight-average molecular weight. $M_{w}^{\frac{1}{2}}$, for polystyrene in benzene ${ }^{82}$, where the values of $z$ have been estimated from observed values of $\alpha_{S}$ using the theories indicated. The data points obtained from the F.o. F.m, and YT theories are seen to fall close to the respective straight lines passing through the origin, indicating that these theories satisfy criterion (2). On the other hand, the $F$ and $P$ equations are seen to lead to nonlinear relations between $z$ and $M^{\frac{1}{2}}$, indicating an inadequacy of equations of the third-power type.

From the above analysis and all previous investigations of this type, we may conclude that combination $Y$ satisfies both criteria (1) and (2). Thus, in what follows, we adopt the YT equation (12) to determine values of $z$ from observed values of $\alpha_{s}$. Very recently, Fujita et al. ${ }^{84}$ have made a similar 
test for polyisobutylene to show that the Flory theory ${ }^{85}$ of $\Psi$ for a uniformdensity sphere model combined with the Fujita-Norisuye theory ${ }^{33}$ of $\alpha_{S}$ is also in good agreement with experiment.

Finally, we note that Berry's analysis has led to estimates of $z$ greater than ours; he has concluded that the Flory-Fisk theory of $\alpha_{S}$ is in good agreement with experiment ${ }^{76}$. Further, we note that the raw data obtained by Kato et $a l .^{86}$ for (anionically prepared) poly- $\alpha$-methylstyrene are different in behavior from all data cited above; for example, Kato's observed values of $\Psi$ reach only 0.2 in good solvents. This difference may be regarded as peculiar to Kato's samples ${ }^{82}$.

\section{Intrinsic viscosities}

Berry ${ }^{77}$ has concluded that for polystyrene the viscosity-radius expansion factor $\alpha_{\eta}$ is not a function of $z$ only, again with the use of the values of $z$ determined following his procedure already mentioned. This result has been interpreted in terms of the draining effect. However, this effect has never been observed for flexible chains at $\Theta$-temperatures, and then there arises the question of whether it actually exists for $z>0$.

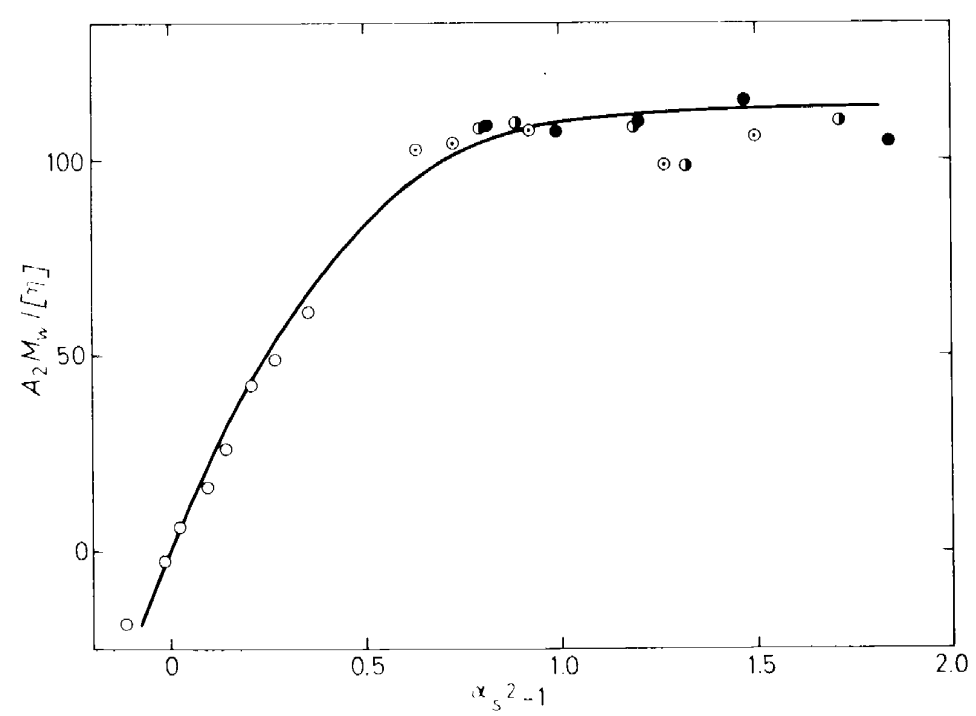

Figure 4. Plots of $A_{2} \mathrm{M}_{w} /[\eta]$ against $\alpha_{s}^{2}-1$ for polystyrene ${ }^{82}$. The symbols have the same significance as those in Figure 2. The curve is an empirical fit to the previous data.

Figure 4 shows plots of $A_{2} M_{w} /[\eta]$ against $\alpha_{S}^{2}-1$ for our polystyrenes ${ }^{82}$, $[\eta]$ being expressed in deciliters per gram. The curve is an empirical fit to all data cited above; the plots may be well represented by a single-composite curve. This suggests that the two-parameter theory does work well for the intrinsic viscosity as well as the equilibrium properties, and therefore that there is no draining effect irrespective of the value of $z$. We note that earlier 


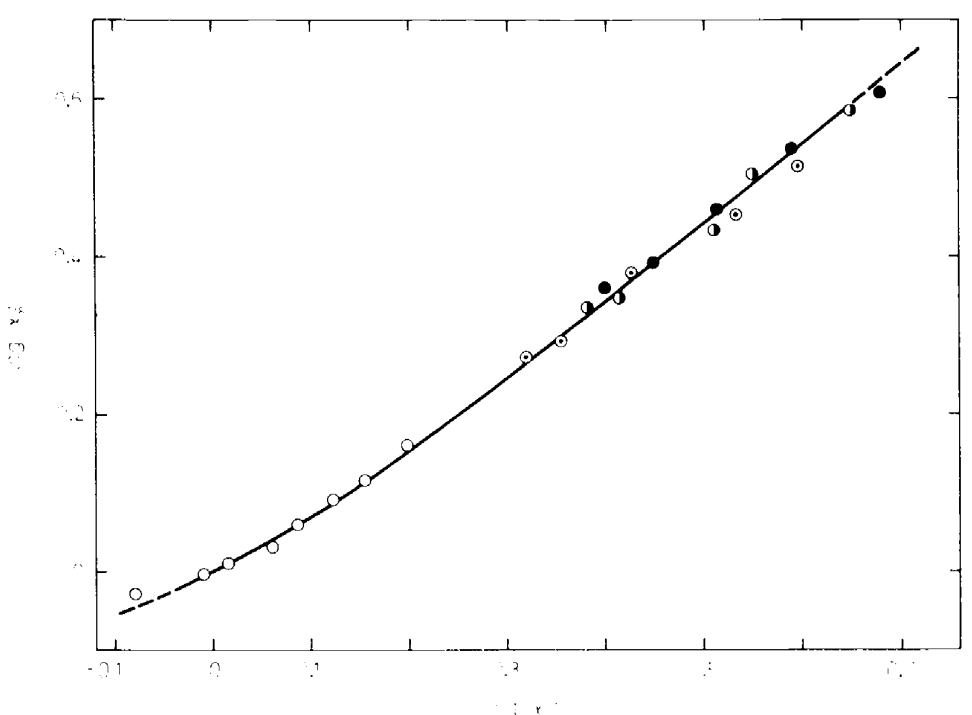

Figure 5. Double logarithmic plots of $x_{\eta}{ }^{3}$ against $x_{s}{ }^{3}$ for polystyrene ${ }^{82}$. The symbols have the same significance as those in Figure' 2 . The curve is an empirical fit to the previous data.

data for the above ratio have been found to scatter remarkably when plotted against $\alpha_{\eta}{ }^{2}-1$, or $\alpha_{s}{ }^{2}-1^{22}$. This is probably due to the inaccuracy of the estimates of $A_{2}$ obtained from the conventional plots.

Whether the draining effect exists or not for $z>0$ may be examined more explicitly as follows. If $\alpha_{\eta}$ is a function of $\alpha_{S}$ only, then $\alpha_{\eta}$ must be a function of $z$ only, since $\alpha_{S}$ is a function of $z$ only. In order to demonstrate this, Fujita et al. ${ }^{79}$ have proposed to plot $\log \alpha_{\eta}{ }^{3}$ against $\log \alpha_{s}{ }^{3}$. Such

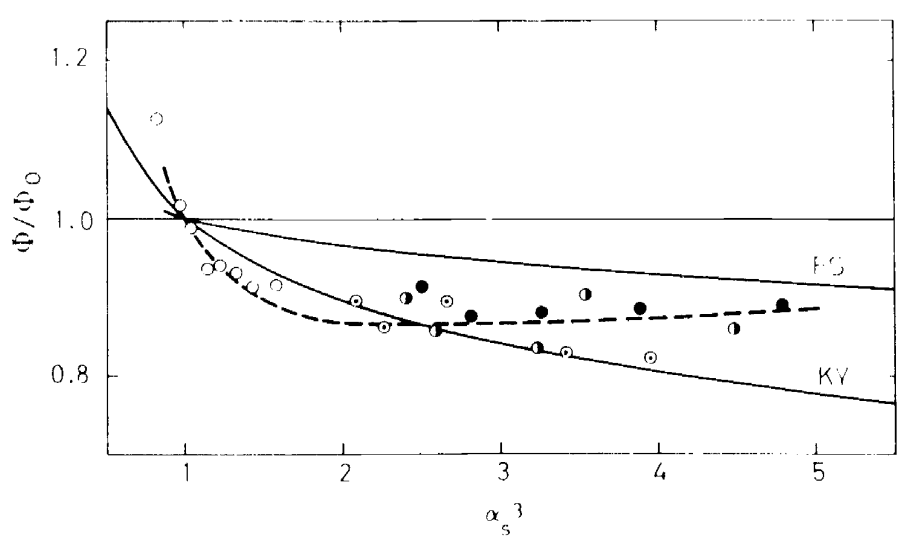

Figure 6. Plots of $\Phi \Phi_{0}$ against $x_{s}{ }^{3}$ for polystyrene ${ }^{42}$. The symbols have the same significance as those in Figure 2. The broken curve is an empirical fit to the previous data. Curve KY: the Kurata Yamakawa theory Curve FS: the Fixman Stidham theory. 
plots can be made without any assumptions, and are explicitly shown for our polystyrenes in Figure 5, where the curve is an empirical fit to all data cited above. The plots form a single-composite curve, and we therefore have support for the earlier conclusion that there is no draining effect for flexible chains irrespective of the value of $z$, as advocated first by Flory ${ }^{2}$. Our problem is then to test approximate two-parameter theories of the intrinsic viscosity. For this purpose, it is convenient to plot $\Phi / \Phi_{0}\left(=\alpha_{\eta}{ }^{3} / \alpha_{s}{ }^{3}\right)$ against $\alpha_{s}{ }^{3}$. Figure 6 shows such plots for our polystyrenes. The broken curve is an empirical fit to the data. The horizontal line, and curves KY and FS represent the values predicted by the Flory-Fox theory, the Kurata-Yamakawa theory $\left(\Phi / \Phi_{0}=\alpha_{S}{ }^{-0.57}\right)^{48}$, and the boson-operator theory of Fixman and Stidham ${ }^{34,35}$, respectively. The observed $\Phi / \Phi_{0}$ is seen to decrease first rapidly and then increase gradually with increasing $\alpha_{s}$. This behavior of $\Phi$ cannot be interpreted by any of the above approximate theories.

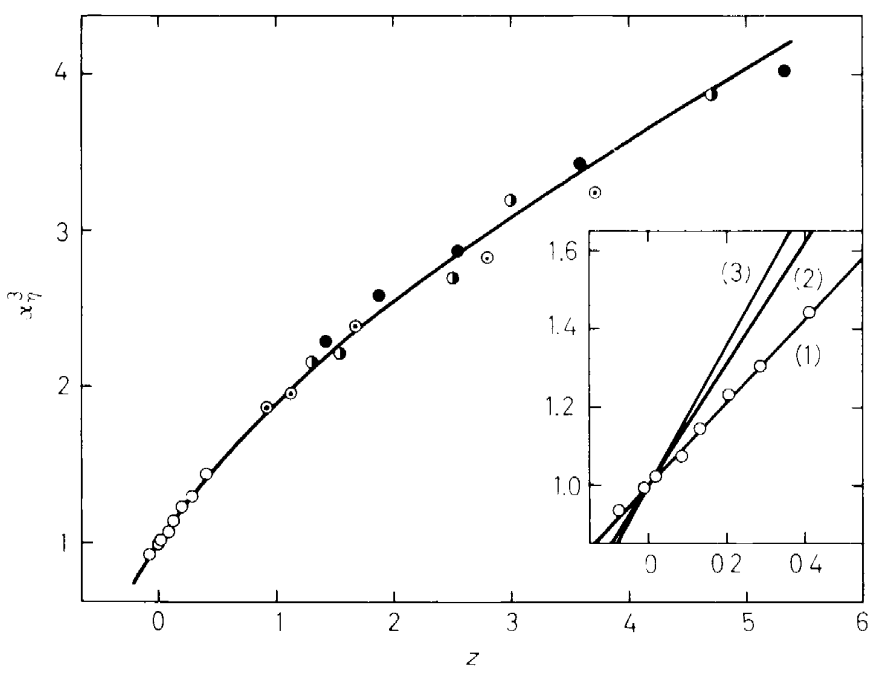

Figure 7. Plots of $\alpha_{\eta}{ }^{3}$ against $z$ for polystyrene ${ }^{82}$, where the values of $z$ were calculated from the values of $\alpha_{s}$ using eyuation 12. The symbols have the same significance as those in Figure 2. The curve is an empirical fit to the previous data. The insert is an enlargement of the region of small $z$; line (1): the first-order perturbation theory of Yamakawa and Tanaka. equation 14 with $C_{1}=1.06$; line (2): the Kurata Yamakawa theory with $C_{1}=1.55$; line (3): the Fixman theory with $\mathrm{C}_{1}=1.80$.

Now we examine the behaviour of $\alpha_{\eta}{ }^{3}$ as a function of $z$. We may determine values of $z$ from observed values of $\alpha_{S}$ using the YT equation (12). as already discussed. In Figure 7 are plotted values of $\alpha_{\eta}{ }^{3}$ against $z$ for our polystyrenes, where the curve is an empirical fit to all data cited above. The insert in the figure is an enlargement of the region of small $z$. Straight lines (1) to (3) represent the first-order perturbation theory predictions (14) with $C_{1}=1.06$ (Yamakawa and Tanaka), $C_{1}=1.55$ (Kurata and Yamakawa), and $C_{1}$ 
$=1.80$ (Fixman). respectively. The new theory of Yamakawa and Tanaka is seen to be in good agreement with experiment. The theory seems to be valid for $z<0.3$, which range is wider than the range $(z<0.15)$ of validity of the first-order perturbation theory of $\alpha_{s}{ }^{3}$.

The curve in Figure 7 has only slight curvature. This characteristic of the plot. which corresponds to the Stockmayer Fixman plot $^{75}$. accounts for why there is a linearity between $[\eta] / M^{\frac{1}{2}}$ and $M^{\frac{1}{2}}$ over a relatively wide range of $M$. Now. except at large $z$. the curve in the figure may be approximated by two straight lines, the equations of which are ar. $^{790}$

$$
\begin{array}{ll}
\alpha_{n}{ }^{3}=1+1.05 z & \text { for } 0<\alpha_{\eta}{ }^{3}<1.6 . \\
x_{n}{ }^{3}=1.05+0.87 z & \text { for } 0<\alpha_{n}{ }^{3}<2.5 .
\end{array}
$$

Since the Stockmayer-Fixman plot is based on the Kurata-Yamakawa equation. $x_{\eta}{ }^{3}=1+1.55 z$. the basic equation for this plot must now be modified (see the next section). We note that the first of equations 16 is approximately valid over a wider range than is the first-order perturbation theory. and therefore that the value of 1.05 for the coelficient of $z$ is not necessarily equal. though close. to the Yamakawa Tanaka value.

\section{BINARY CLUSTER INTEGRALS}

Having established the equations for $\alpha_{S}$ and $\alpha_{\eta}$. we can now estimate values of the parameter $z$. or the interaction parameter $B$ defined by

$$
B=(n / M)^{2} \beta \text {. }
$$

by the use of these equations. As already noted and also as seen from equation 17. an analysis of the binary cluster integral $\beta$ itself requires the assumption of the size of a segment. Throughout the remainder of this paper, we consider the binary cluster integral per monomeric unit. for convenience. We first summarize practical methods of determining $\beta$, and then attempt a theoretical interpretation of the values of $\beta$ for several systems.

\section{Experimental determination}

There are two methods. One is based on equation 12. We first determine values of $z$ from observed values of $\alpha_{S}$ for various molecular weights using equation 12 . and then plot the values of $z$ against $M^{\frac{1}{2}}$. as in Figure 3 . We can determine $\beta$ from the slope of this plot by the use of equations 3 and 17 .

The other is an indirect method applying the Stockmayer Fixman plot. Since we have established the new empirical equations 16 for $x_{\eta}$, the basic equations for this plot must be modified as follows.

$$
\begin{array}{ll}
{[\eta] M^{\frac{1}{2}}=K+0.346 \Phi_{0} B M^{\frac{1}{2}}} & \text { for } 0<\alpha_{\eta}{ }^{3}<1.6 . \\
{[\eta] / M^{\frac{1}{2}}=1.05 K+0.287 \Phi_{0} B M^{\frac{1}{2}}} & \text { for } 0<\alpha_{\eta}{ }^{3}<2.5 .
\end{array}
$$

where $K=[\eta]_{\theta} / M^{\frac{1}{2}}$ with $[\eta]_{\theta}$ the intrinsic viscosity of unperturbed chains, and we may assume $\Phi_{0}=2.5 \times 10^{2.3}(\mathrm{cgs})$. Thus we can determine $\beta$ from the slope of the plot by the use of equations 17 and 18 . According to equations 18. values of $[\eta] / M_{w}{ }^{\frac{1}{4}}$ for our polystyrenes are plotted against $M_{w}{ }^{1}$ in Figure 8 . The two thin horizontal lines in the figure indicate the upper bounds below 


\section{EXCLUDED VOLUME EFFECTS AND BINARY CLUSTER INTEGRALS}

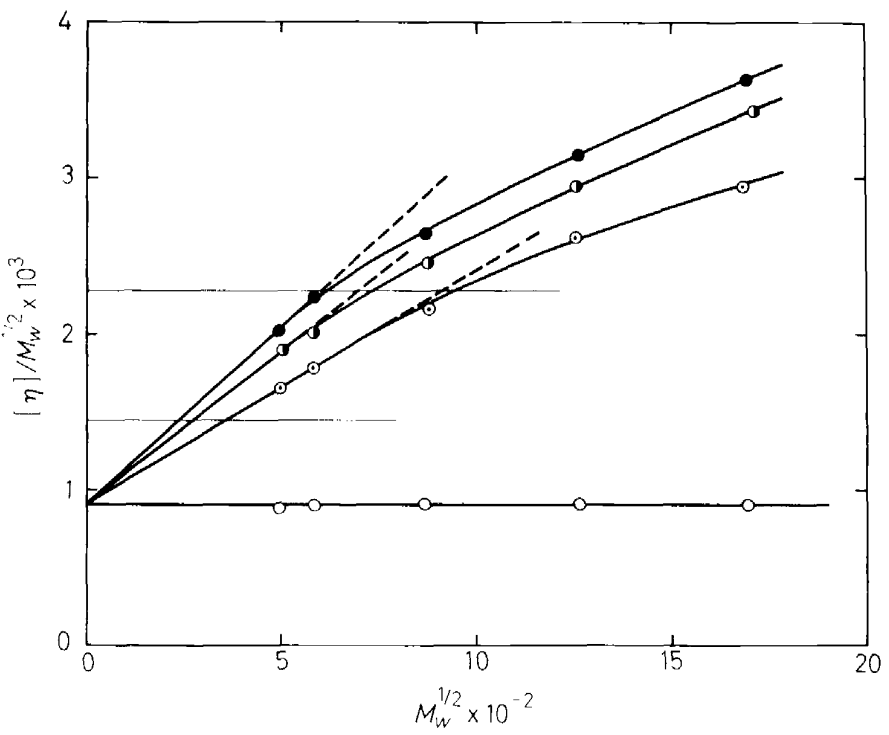

Figure 8. Stockmayer-Fixman plots for polystyrene: $\mathrm{O}$, in benzene at $30^{\circ} \mathrm{C}:(1)$ in toluene at $30^{\circ} \mathrm{C}: \odot$. in dichloroethane at $30^{\circ} \mathrm{C}: \bigcirc$, in cyclohexane at the $\Theta$-temperature ${ }^{82}$. The broken lines are the initial tangents. The two thin horizontal lines indicate the upper bounds given in equations 18 .

which equations 18 are applicable, corresponding to $[\eta] / K M_{w}^{\frac{1}{2}}=1.6$ and 2.5 , respectively. For good solvent systems, extrapolations to $M_{w}=0$ have been carried out to give the same intercept as that in the $\Theta$-solvent, neglecting the minor difference between $K$ and $1.05 K$. The broken lines in the figure indicate the tangents to those parts of the curves which may be regarded as linear. Values of $B$ and hence $\beta$, can be obtained from the slopes of these

Table 2. Values of $\beta$ per monomeric unit estimated from expansion factors and intrinsic viscosities

\begin{tabular}{|c|c|c|c|c|}
\hline \multirow{2}{*}{ Polymer } & \multirow{2}{*}{ Solvent } & \multirow{2}{*}{ Temp. ${ }^{\circ} \mathrm{C}$} & \multicolumn{2}{|c|}{$\beta \times 10^{24} \mathrm{~cm}^{3}$} \\
\hline & & & From $\alpha_{s}$ & From $[\eta]$ \\
\hline \multirow{3}{*}{$\begin{array}{l}\text { Polystyrene }^{82} \\
\text { Poly-p-methylstyrene }\end{array}$} & Dichloroethane & 30 & 24.4 & 22.9 \\
\hline & Toluene & 30 & 40.4 & 38.0 \\
\hline & Methyl ethyl ketone & 30 & 12.4 & 10.3 \\
\hline \multirow[t]{3}{*}{ Poly-p-chlorostyrene ${ }^{87,88}$} & Monochlorobenzene & 30 & 20.0 & 22.9 \\
\hline & Toluene & 30 & 9.90 & 9.78 \\
\hline & Benzene & 30 & 5.69 & 5.86 \\
\hline \multirow[t]{2}{*}{ Poly-p-bromostyrene $\mathrm{e}^{81,87}$} & Monochlorobenzene & 30 & 27.8 & 27.3 \\
\hline & Toluene & 30 & 1.84 & 1.94 \\
\hline \multirow[t]{2}{*}{ Polychloroprene ${ }^{78.79}$} & Carbon tetrachloride & 25 & 21.0 & 23.5 \\
\hline & $n$-Butyl acetate & 25 & 8.99 & 8.77 \\
\hline
\end{tabular}


broken lines using the second of equations 18. (If a linear part of the plot lies below the lower horizontal line, the first of equations 18 is to be used.)

Table 2 summarizes values of $\beta$ thus obtained for several polymers in various solvents ${ }^{78-82,87.88}$. It is seen that there is good agreement between the values determined by the two methods. The second indirect method is useful when there are no available light-scattering data.

\section{Theoretical interpretation}

As seen from the definition of $\beta$, it is closely related to the second virial coefficient for segments, or monomeric units. Consider now a hypothetical solution such that the solute molecules are monomeric units which would be obtained by cutting the parent chain. The second virial coefficient $A_{2}{ }^{0}$ for this solution may be related to $\beta$ by the equation.

$$
A_{2}{ }^{0}=N_{A} \beta / 2 m^{2} \text {, }
$$

where $m$ is the molecular weight of the monomeric unit. We note that $A_{2}{ }^{0}$ is just the single-contact term of the second virial coefficient for the polymers. Thus $\beta$ can be computed by an evaluation either of the free energy of mixing or of the pair correlation function for solutes in very dilute solutions composed of small molecules. Yamakawa et al. ${ }^{89}$ have evaluated the excess binary cluster integral for polar polymers by the second approach, a comparison of theory with experiment having been made for poly-p-halostyrenes. Very recently. Yamakawa and Fujiii ${ }^{90}$ have attempted a simple analysis by the first approach, which we briefly describe here.

Yamakawa and Fujii have adopted the smoothed-potential cell model of Prigogine ${ }^{91}$ to evaluate the free energy of mixing. For this model, the configurational partition function $Z$ for the solution (of small molecules) may be written in the form.

$$
Z=\text { const. } Z_{\mathrm{c}}\left(v^{\frac{1}{4}}-v^{* \frac{1}{4}}\right)^{3 N} \exp \left(-E_{0} / k T\right),
$$

where $Z_{\mathrm{c}}$ denotes the combinatorial factor, $N$ is the number of all molecules in the solution, $v$ is the average molecular volume, $v^{*}$ is its hard core volume. and $E_{0}$ is the average intermolecular energy. It is now known that real liquid mixtures may be well represented by van der Waals liquid models ${ }^{92.93}$, as defined by $E_{0}=$ const. $v^{-1}$ and used by Flory and co-workers ${ }^{94.97}$. However, we must assume Lennard-Jones liquid models for our purpose, since we are considering the hypothetical solution and equation of state data for the solute monomeric units are not available. Although such models are less satisfactory, the procedure adopted has been shown to be good enough to draw an important conclusion as a first approximation ${ }^{90}$.

Then, the thermodynamic properties, such as $A_{2}{ }^{0}$, of the solution may be finally expressed in terms of the Lennard-Jones force constants, $\sigma_{i j}$ and $\varepsilon_{i j}$ $(i, j=1,2)$, for components $i$ and $j$. Values of $\sigma_{11}$ and $\varepsilon_{11}$ for various solvents are now available ${ }^{98}$, and $\sigma_{12}$ and $\varepsilon_{12}$ may be determined by the use of the Lorentz-Berthelot rules. The constants $\sigma_{22}$ and $\varepsilon_{22}$ for monomeric units have been determined to give close agreement between observed and calculated values of $\beta$ in $\Theta$-solvent systems ${ }^{90}$. In Tables 3 and 4, are given 
EXCLUDED VOLUME EFFECTS AND BINARY CLUSTER INTEGRALS

Table 3. Observed and calculated values of $\beta$ for monomeric units of polystyrene

\begin{tabular}{|c|c|c|c|}
\hline \multirow{2}{*}{ Solvent } & \multirow{2}{*}{ Temp. ${ }^{\circ} \mathrm{C}$} & \multicolumn{2}{|c|}{$\beta \times 10^{24} \mathrm{~cm}^{3}$} \\
\hline & & Obs. $^{a}$ & Calc. \\
\hline \multirow[t]{4}{*}{ Cyclohexane } & 32 & -1.08 & -8.61 \\
\hline & 35 & 0.28 & 0.591 \\
\hline & 40 & 1.88 & 15.8 \\
\hline & 50 & 4.10 & 45.7 \\
\hline \multirow[t]{3}{*}{ Toluene } & 30 & 31.2 & 275 \\
\hline & 40 & - & 290 \\
\hline & 50 & $\ldots$ & 304 \\
\hline \multirow[t]{3}{*}{ Benzene } & 30 & 35.6 & 294 \\
\hline & 40 & - & 335 \\
\hline & 50 & - & 373 \\
\hline
\end{tabular}

"See the text and ref. 82 .

observed and calculated values of $\beta$ for polystyrene and polyisobutylene, respectively. The observed values of $\beta$ for polystyrene have been obtained from $\alpha_{S}{ }^{82}$, and those for polyisobutylene have been obtained from $[\eta]^{99,100}$ except the value in cyclohexane at $25^{\prime \prime} \mathrm{C}$ determined from $\alpha_{S}{ }^{84}$.

It is seen that the present model can interpret qualitatively the behaviour of $\beta$. That is, there have been obtained values of $\beta$ which are small and appreciably dependent on temperature in $\Theta$-solvents, and values which are large and almost independent of temperature in good solvents. However, the calculated values of $\beta$ are an order of magnitude greater than the observed values. For comparison, the corresponding observed values for several

Table 4. Observed and calculated values of $\beta$ for monomeric units of polyisobutylene

\begin{tabular}{|c|c|c|c|}
\hline \multirow{2}{*}{ Solvent } & \multirow{2}{*}{ Temp. "C } & \multicolumn{2}{|c|}{$\beta \times 10^{24} \mathrm{~cm}^{3}$} \\
\hline & & Obs. & Calc. \\
\hline \multirow{4}{*}{ Benzene } & 22 & $-0.681^{a}$ & -7.54 \\
\hline & 25 & $0.191^{a}$ & 1.62 \\
\hline & 35 & $1.50^{a}$ & 32.3 \\
\hline & 50 & $3.51^{a}$ & 73.7 \\
\hline \multirow{3}{*}{ Toluene } & 20 & $3.80^{a}$ & 166 \\
\hline & 30 & - & 191 \\
\hline & 40 & $5.13^{a}$ & 221 \\
\hline \multirow[t]{3}{*}{ Cyclohexane } & 25 & $16.0^{b}$ & 677 \\
\hline & 30 & $19.8^{c}$ & 629 \\
\hline & 40 & - & 565 \\
\hline
\end{tabular}

"See the text and ref. 99.

"See the text and ref. 84

' See the text and ref. 100. 
systems composed of real small molecules ${ }^{101.102}$ are given in Table 5. It is important to observe that the calculated values of $\beta$ for polymer segments are the same order of magnitude as the observed values of $\beta$ for real small molecules. This is true, because we have calculated $\beta$ for the (hypothetical) solutions of small molecules. In this connection. we note that the theoretical $\beta$ for polystyrene in toluene is equal to the molecular volume of toluene,

Table 5. Observed values of $\beta$ for binary mixtures of small molecules

\begin{tabular}{llcc}
\hline \multicolumn{1}{c}{ Solute } & \multicolumn{1}{c}{ Solvent } & Temp. $\mathrm{C} \beta \times 10^{24} \mathrm{~cm}^{3}$ \\
\hline Benzene $^{101}$ & Carbon tetrachloride & 25 & 100 \\
& Cyclohexane & 40 & 102 \\
& & 25 & -70.2 \\
Carbon tetrachloride $^{101}$ & Benzene & 40 & -39.8 \\
& & 25 & 140 \\
& Cyclohexane & 40 & 142 \\
Cyclohexane $^{101}$ & & 25 & 111 \\
& Benzene & 40 & 114 \\
& & 25 & 19.4 \\
Chloroform $^{102}$ & Carbon tetrachloride & 40 & 40.8 \\
Ethanol $^{102}$ & & 25 & 158 \\
Acetone $^{102}$ & Acetone & 30 & 164 \\
& Chloroform & 35 & 298 \\
& Chloroform & 35.17 & 470 \\
\hline
\end{tabular}

e.g. $178 \times 10^{-24} \mathrm{~cm}^{3}$ at $30 \mathrm{C}$. if the hypothetical solution is considered an isotope mixture in a first approximation ${ }^{3.52}$. Thus, observed values of $\beta$ for polymer segments are definitely an order of magnitude smaller than the values expected from second virial coefficients for small molecules. This difference may be regarded as arising from the fact that the degrees of freedom. translational and rotational, of polymer segments bound in the chain are lower than those of free small molecules. At present, it is difficult to interpret completely interactions between polymer segments in dilute solution on the molecular and atomic levels, that is, in terms of intermolecular forces.

\section{CONCLUSIONS}

We have critically summarized recent advances in the field of dilute polymer solutions. We now tend to believe that the asymptotic solution to the excluded volume problem obeys the fifth-power law rather than the third-power law, though there have been many controversies. In the development of the theory applicable over the range experimentally accessible, emphasis has been focused on its self-consistency. This has led to reliable estimates of the binary cluster integral. It has been pointed out that a difficulty arises in the interpretation of the interaction between segments on 


\section{EXCLUDED VOLUME EFFECTS AND BINARY CLUSTER INTEGRALS}

the molecular level. The asymptotic solution for the expansion factor and the many-body problem in the nonequilibrium theory require further investigations.

\section{ACKNOWLEDGMENTS}

The author wishes to thank the Scientific and Organizing Committee of the 9th I.U.P.A.C. Microsymposium for inviting his lecture. He also thanks his students and collaborators for their assistance in the work summarized in this paper.

\section{REFERENCES}

1 P. J. Flory, J. Chem. Phys. 17, 303 (1949).

${ }^{2}$ P. J. Flory, Principles of Polymer Chemistry. Cornell University Press, Ithaca. New York (1953).

${ }^{3}$ H. Yamakawa, Modern Theory of Polymer Solutions. Harper \& Row, New York (1971),

${ }^{4}$ M. V. Volkenstein, Configurational Statistics of Polymeric Chains. Interscience, New York (1963).

${ }_{5}$ T. M. Birshtein and O. B. Ptitsyn, Conformations of Macromolecules. Interscience, New York (1966).

${ }^{\circ}$ P. J. Flory, Statistical Mechanics of Chain Molecules. Interscience, New York (1969).

${ }^{7 a}$ E. Teramoto, Busseiron Kenkyu 39, 1 (1951).

${ }^{b}$ E. Teramoto, Busseiron Kenkyu 40, 18 (1951).

' E. Teramoto, Busseiron Kenkyu 41, 14 (1951).

${ }^{8}$ M. Yamamoto, Busseiron Kenkyu 44, 36 (1951).

9 B. H. Zimm, W. H. Stockmayer and M. Fixman, J. Chem. Phys. 21, 1716 (1953).

10 M. Fixman, J. Chem. Phys. 23, 1656 (1955).

11 M. Kurata, H. Yamakawa and E. Teramoto, J. Chem. Phys. 28, 785 (1958).

12 H. Yamakawa, A. Aoki and G. Tanaka, J. Chem. Phys. 45, 1938 (1966).

${ }^{13}$ H. Yamakawa and G. Tanaka, J. Chem. Phys. 47, 3991 (1967).

14 S. F. Edwards, Proc. Phys. Soc. (London) 85, 613 (1965).

15 H. Reiss. J. Chem. Phys. 47, 186 (1967).

16 J. G. Curro and P. J. Blatz, J. Chem. Phys. 48, 2832 (1968).

17 R. Yeh and A. Isihara, J. Chem. Phys. 51, 1215 (1969).

18 H. Yamakawa, J. Chem. Phys. 48, 3845 (1968).

19 H. Yamakawa, J. Chem. Phys. 54, 2484 (1971).

20 K. F. Freed, J. Chem. Phys. 55, 3910 (1971).

21 M. Kurata, W. H. Stockmayer and A. Roig, J. Chem. Phys. 33. 151 (1960).

${ }_{22}$ M. Kurata and W. H. Stockmayer, Fortschr. Hochpolymer. Forsch. 3, 196 (1963).

23 J. Naghizadeh, J. Chem. Phys. 48, 1961 (1968).

24 J. G. Curro, P. J. Blatz and C. J. Pings, J. Chem. Phys. 50, 2199 (1969).

25 H. Fujita, K. Okita and T. Norisuye, J. Chem. Phys. 47, 2723 (1967).

${ }^{26}$ M. Kurata. J. Polymer Sci. A2, 6, 1607 (1968).

27 Z. Alexandrowicz, J. Chem. Phys. 49, 1599 (1968).

${ }^{28}$ P. J. Flory and S. Fisk, J. Chem. Phys. 44, 2243 (1966).

${ }^{29}$ M. Fixman, J. Chem. Phys. 36, 306 (1962).

${ }^{30 a}$ W. C. Forsman and R. E. Hughes, J. Chem. Phys. 38, 2118 (1963).

${ }^{b}$ W. C. Forsman and R. E. Hughes, J. Chem. Phys. 42, 2829 (1965).

${ }^{31}$ R. F. Hoffman and W. C. Forsman, J. Chem. Phys. 50, 2316 (1969).

32 R. Koyama, J. Phys. Soc. Japan 24, 580 (1968).

${ }^{33}$ H. Fujita and T. Norisuye, J. Chem. Phys. 52, 1115 (1970).

${ }^{34 a}$ M. Fixman, J. Chem. Phys. 45, 785 (1966).

${ }^{b}$ M. Fixman, J. Chem. Phys. 45, 793 (1966).

35 H. D. Stidham and M. Fixman, J. Chem. Phys. 48, 3092 (1968).

36 M. Fixman, J. Chem. Phys. 36, 3123 (1962). 


\section{HIROMI YAMAKAWA}

37 O. B. Ptitsyn, Vysokomolekul. Soedin. 3, 1673 (1961).

38 A. K. Kron and O. B. Ptitsyn, Vysokomolekul. Soedin. A, 9, 759 (1967).

39 K. Suzuki, Bull. Chem. Soc. Japan 41, 538 (1968).

40 Z. Alexandrowicz, J. Chem. Phys. 51, 561 (1969).

41 C. Domb, J. Chem. Phys. 38, 2957 (1963).

42 C. Domb, Advan. Chem. Phys. 15, 229 (1969).

43 V. A. Bloomfield and D. S. McKenzie, J. Chem. Phys. 52, 628 (1970).

44 M. Kurata. M. Fukatsu, H. Sotobayashi and H. Yamakawa, J. Chem. Phys. 41, 139 (1964).

45 Y. Tagami and E. F. Casassa, J. Chem. Phys. 50, 2206 (1969).

46 B. H. Zimm, J. Chem. Phys. 14, 164 (1946).

47 A. C. Albrecht, J. Chem. Phys. 27, 1002 (1957).

48 M. Kurata and H. Yamakawa, J. Chem. Phys. 29, 311 (1958).

49 H. Yamakawa, J. Chem. Phys. 48, 2103 (1968).

50 P. J. Flory and W. R. Krigbaum. J. Chem. Phys. 18, 1086 (1950).

51 T. A. Orofino and P. J. Flory. J. Chem. Phys. 26, 1067 (1957).

52 W. H. Stock mayer, Makromol. Chem. 35, 54 (1960).

53 P. J. Flory and T. G. Fox. Jr., J. Am. Chem. Soc. 73, 1904 (1951).

54 J. G. Kirkwood and J. Riseman, J. Chem. Phys. 16, 565 (1948).

55a J. G. Kirkwood, Rec. Trav. Chim. 68, 649 (1949).

${ }^{b}$ J. G. Kirkwood, J. Polymer Sci. 12, 1 (1954).

56 P. E. Rouse, Jr., J. Chem. Phy's. 21, 1272 (1953).

57 F. Bueche, J. Chem. Phys. 22, 603 (1954).

58 B. H. Zimm, J. Chem. Phys. 24. 269 (1956).

59 R. E. De Wames, W. F. Hall and M. C. Shen, J. Chem. Phys. 46, 2782 (1967).

60 R. Zwanzig, J. Kiefer and G. H. Weiss, Proc. Nat. Acad. Sci. U.S. 60, 381 (1968).

61 A. Perico and C. Rossi, J, Chem. Phys, 53, 1217 (1970).

62 G. B. Thurston and J. D. Morrison, Polymer 10, 421 (1969).

63 J. Rotne and S. Prager, J. Chem. Phys. 50, 4831 (1969).

${ }^{64}$ H. Yamakawa, J. Chem. Phys. 53, 436 (1970).

65 H. Yamakawa and G. Tanaka, J. Chem. Phys. In press.

$66 \mathrm{H}$. Yamakawa and J. Yamaki, J. Chem. Phys.. In press.

67 J. E. Hearst, J. Chem. Phys. 40, 1506 (1964).

68 J. E. Hearst and Y. Tagami, J. Chem. Phys. 42. 4149 (1965),

${ }^{69}$ S. F. Edwards and M. A. Oliver, J. Phys. A. 4. 1 (1971).

${ }^{70 a}$ P. L. Auer and C. S. Gardner, J. Chem. Phys. 23, 1545 (1955).

${ }^{b}$ P. L. Auer and C. S. Gardner, J. Chem. Phys. 23, 1546 (1955).

71 J. E. Hearst, J. Chem. Phys. 37, 2547 (1962).

72 M. Fixman, J. Chem. Phys. 42, 3831 (1965).

${ }^{73 a} \mathrm{C}$. W. Pyun and M. Fixman, J. Chem. Phys. 42, 3838 (1965).

${ }^{b} \mathrm{C}$. W. Pyun and M. Fixman, J. Chem. Phys. 44, 2107 (1966).

74 H. Yamakawa and G. Tanaka, J. Chem. Phys. 55. 3188 (1971).

75 W. H. Stockmayer and M. Fixman, J. Polymer Sci. C1, 137(1963).

${ }^{76}$ G. C. Berry, J. Chem. Phys. 44, 4550 (1966).

77 G. C. Berry, J. Chem. Phys. 46, 1338 (1967).

${ }_{78}$ T. Norisuye, K. Kawahara, A. Teramoto and H. Fujita, J. Chem. Phys, 49, 4330 (1968).

79 K. Kawahara. T. Norisuye and H. Fujita, J. Chem. Phys, 49, 4339 (1968).

${ }^{80}$ G. Tanaka, S. Imai and H. Yamakawa, J. Chem. Phys. 52, 2639 (1970).

81 K. Takashima, G. Tanaka and H. Yamakawa, Polymer J. 2. 245 (1971).

82 A. Yamamoto, M. Fujii, G. Tanaka and H. Yamakawa, Polymer J. 2, 799 (1971).

83 E. F. Casassa and H. Markovitz, J. Chem. Phys. 29, 493 (1958).

${ }^{84}$ T. Matsumoto, N. Nishioka and H. Fujita, J. Polymer Sci. A2. 10, 23 (1972).

85 P. J. Flory. J, Chem. Phys. 13, 453 (1945).

86 T. Kato, K. Miyaso. I. Noda. T. Fujimoto and M. Nagasawa, Macromolecules 3. 777 (1970).

87 Y. Noguchi, A. Aoki, G. Tanaka and H. Yamakawa, J. Chem. Phys. 52, 2651 (1970).

${ }_{88}$ M. Fujii and H. Yamakawa. Unpublished data.

${ }^{89}$ H. Yamakawa, S. A. Rice, R. Corneliussen and L. Kotin, J. Chem. Phys. 38, 1759 (1963).

90 H. Yamakawa and M. Fujii, J. Chem. Phys. To be published.

91 I. Prigogine, The Molecular Theory of Solutions, North-Holland Publishing Co., Amsterdam (1957). 


\section{EXCLUDED VOLUME EFFECTS AND BINARY CLUSTER INTEGRALS}

92 J. H. Hildebrand and R. L. Scott, Regular Solutions, Prentice-Hall, Englewood Cliffs, N.J., U.S.A. (1962).

93 J. S. Rowlinson, Disc. Faraday Soc. 49, 30 (1970).

${ }^{94 a}$ P. J. Flory, R. A. Orwoll and A. Vrij, J. Am. Chem. Soc. 86, 3507 (1964).

${ }^{b}$ P. J. Flory, R. A. Orwoll and A. Vrij, J. Am. Chem. Soc. 86,3515 (1964).

95 P. J. Flory and A. Abe, J. Am. Chem. Soc. 86, 3563 (1964).

96 P. J. Flory, J. Am. Chem. Soc. 87, 1833 (1965).

${ }^{97}$ A. Abe and P. J. Flory, J. Am. Chem. Soc. 87, 1838 (1965).

98 J. O. Hirschfelder, C. F. Curtiss and R. B. Bird, Molecular Theory of Gases and Liquids. John Wiley \& Sons, New York (1964).

99 T. G Fox, Jr. and P. J. Flory, J. Am. Chem. Soc. 73, 1909 (1951).

100 W. R. Krigbaum and P. J. Flory, J. Polymer Sci. 11, 37 (1953).

101 J. R. Goates, R. J. Sullivan and J. Bevan, J. Phys. Chem. 63, 589 (1959).

102 H. C. Carlson and P. Colbern, Ind. Eng. Chem. 34, 581 (1942). 